Communication

\title{
Reducing Further Spread of COVID-19 in Taiwan by Containment of Overseas Immigration, Hospital Cluster Infection Control, and Mass Gathering Warnings
}

\author{
Yang Chih-Ching ${ }^{1,2,3}$, Wei Cheng-Yu ${ }^{3}$ \\ ${ }^{1}$ School of Life Science, National Taiwan Normal University, Taipei, Taiwan \\ ${ }^{2}$ Center for General Education, Mackay College of Medical, Nursing, and Management, Taipei, Taiwan \\ ${ }^{3}$ Office of Public Relation of Ministry of Health and Welfare, Taipei, Taiwan
}

Email address:

mdyangcc@mohw.gov.tw (Yang Chih-Ching), easytalkgamily888@icloud.com (Wei Cheng-Yu)

\section{To cite this article:}

Yang Chihching, Wei Chengyu. Reducing Further Spread of COVID-19 in Taiwan by Containment of Overseas Immigration, Hospital Cluster Infection Control, and Mass Gathering Warnings. American Journal of Internal Medicine. Vol. 8, No. 4, 2020, pp. 192-196.

doi: 10.11648/j.ajim.20200804.18

Received: June 1, 2020; Accepted: June 18, 2020; Published: August 10, 2020

\begin{abstract}
The experience that Taiwan gained from the Severe Acute Respiratory Syndrome (SARS) epidemic in 2003 helped in the development of early preparation and response to COVID-19 outbreak. Measures including isolation, quarantine, and social distancing have been promptly taken to flatten the epidemic progress curve in the first 50 days since Dec. 31, 2019. In the second stage, the focus of epidemic prevention in Taiwan has shifted gradually from "containment" to "disaster reduction". "Social distancing" is now a strategy for reducing transmission and "flattening the curve" with respect to cases entering the health care system. The further spread of COVID-19 in Taiwan was alleviated by control of movement in and out of the country, hospital clustering infection control, and the prevention of mass gatherings successfully. We already delayed the peak, therefore exports has become easier and we could help other countries with the necessary supply of materials. Overall, the performance of Taiwan's COVID-19 epidemic infection control was ranked first place among the 23 OECD countries. Most of the confirmed patients were aged among 20 to 40 years old, which accounted for $60 \%$ of total confirmed cases, with a total mortality rate of about $1.4 \%$. The median time from the onset of symptoms to the release from isolation was 30 days. Due to this severe economic impact from COVID-19 epidemic, Taiwan government quickly responded with the three major principles of "epidemic prevention, bail-out, and revitalization", in order to reduce negative impact on many industries and corporations.
\end{abstract}

Keywords: COVID-19 Outbreak, Containment of Immigration, Hospital Cluster Infection, Mass Gathering

\section{Introduction}

The WHO classified the COVID-19 outbreak as a pandemic on March 12. This pandemic will be a long-term dynamic event that will require continuous strategy development and problem solving. The experience that Taiwan gained from the Severe Acute Respiratory Syndrome (SARS) epidemic in 2003 helped in the development of early preparation and response to this new novel threat. Measures including isolation, quarantine, and social distancing have been promptly taken to flatten the epidemic progress curve
[1]. A sound public health network, support from the healthcare industry, cross-departmental collaboration, and advanced information technology capacity has helped Taiwan delay the first stage [2]. Community transmission was contained by the leveraging of experience from the SARS outbreak and prevalent public awareness. In the second stage, the focus of epidemic prevention in Taiwan has shifted gradually from "containment" to "disaster reduction". "Social distancing" is now a strategy for reducing transmission and "flattening the curve" with respect to cases entering the health care system. [3] The major strategy has been suppression of the epidemic peak and the delay of 
outbreak time. Taiwan is not the only country that has adopted this strategy of flattening the curve; Europe and now the United States, are using a similar approach.

There is now an urgent need for an increase in the production of epidemic prevention supplies such as personal protection equipment (PPE), fast screening tests, therapeutic drugs, as well as an acceleration of vaccine research and development. If we can delay the peak there will be more time for these. Exports will become easier and we can help other countries with the necessary supply of materials [4]

The further spread of COVID-19 in Taiwan can be alleviated by control of movement in and out of the country, hospital clustering infection control, and the prevention of mass gatherings. (See Figure 1)

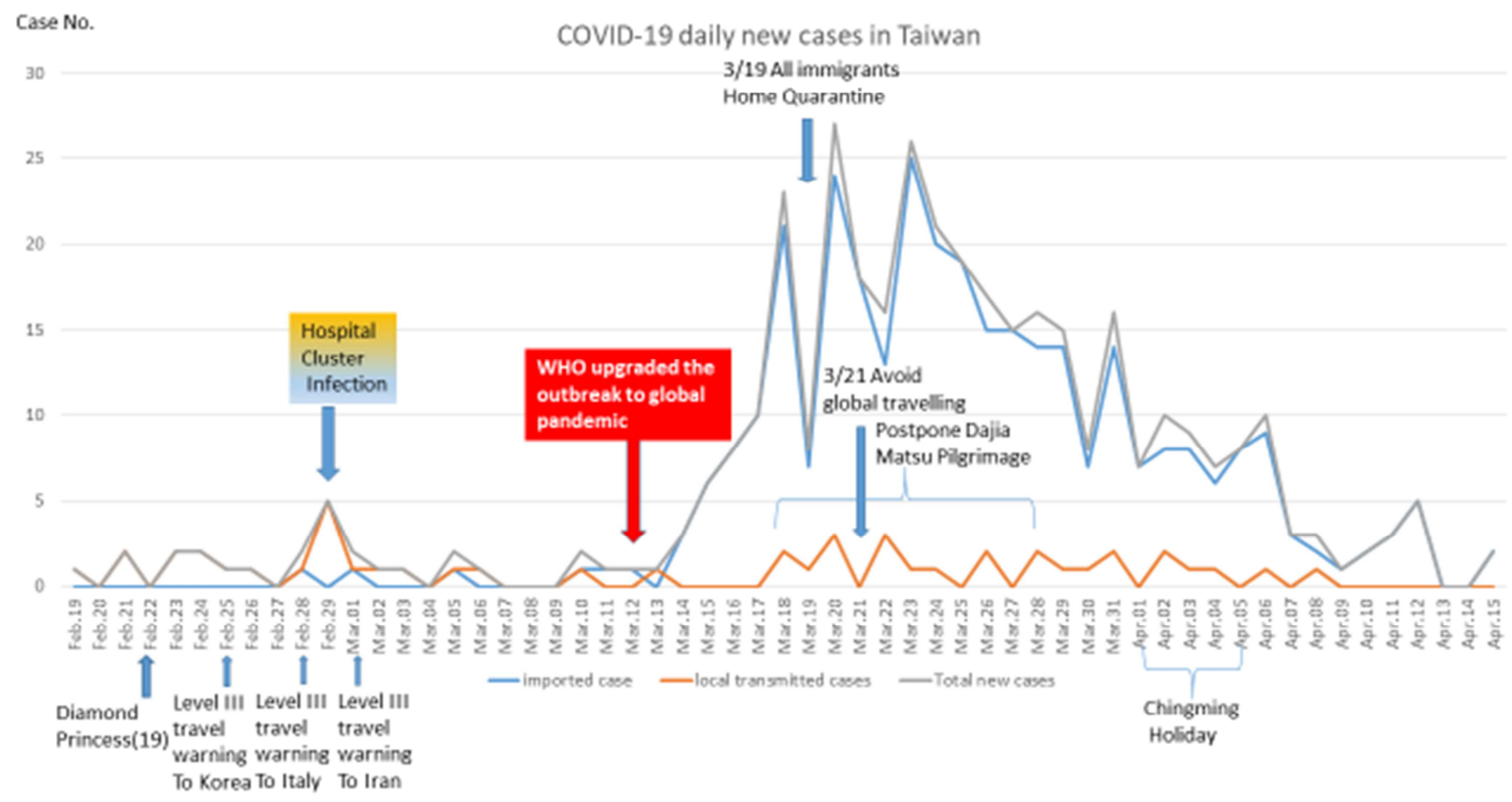

Figure 1. Timeline of daily new cases during Feb. 19 to Apr. 15 of COVID-19 in Taiwan. Blue-imported cases; orange-local transmitted cases; and graydaily reported new cases in Taiwan. Information collected from Taiwan CDC. MM.

\section{Material and Method}

\subsection{Containment of Immigration}

Most of the first wave of the epidemic in the Spring was successfully blocked and now the second wave associated with Global migration, is receiving attention. The most serious thing to be worried about in this respect are asymptomatic or minimally symptomatic carriers [5-8]. All people coming into Taiwan (after March 19) are being quarantined for 14 days and about 75,000 people are being examined at present. In addition, because of the COVID-19 epidemic, many people have been isolated and quarantined. In order to protect the rights of those people to ask for medical service, Taiwan government has recently allowed medical institutions to provide remote healthcare to those people who were isolated or quarantined, with "Cloud X-ray interpretation technology", "Real-time temperature monitoring patch", "ENT and Eye scope", and the mobile medical platform. This project has been piloted in governmental hospitals, and it has been gradually extended to clinics and designated group quarantine site. Cloud X-ray interpretation technology can evaluate abnormal X-rays through AI algorithm to assist physicians in finding out patients with pneumonia more quickly. In the near future, if people have a fever or any physical discomfort, they can go to the clinic for a preliminary examination, then the tentative diagnosis and chest X-ray image will be preliminarily interpreted through the cloud AI. Besides, these data will be sent to an infectious specialist for a remote consultation in real time, to provide further suggestion to the clinic physician. This telemedicine model could be expected to effectively reduce the infection spreading risk on hospital visits.

\subsection{Hospital Clustering Infection}

Taiwan paid a heavy price in the battle against SARS, but learned valuable lessons. In 2003, a large-scale nosocomial infection broke out in the Taipei Municipal Hoping hospital (TMHH) and the hospital had to be closed down. The same thing happened at the National Taiwan University Hospital (NTUH) but both these events were handled properly and although several NTUH nurses were infected, no one died.

This time the source of the infection was found immediately and successfully stopped.

The outbreak of the COVID-19 nosocomial infection in Chang Gung Memorial Hospital was confirmed on Feb 29, and by March 21, the situation was well under control and 
there was no spread to the community. During these 21 days, the health unit closed the inpatient ward and moved all the patients out. The ward staff were isolated and screened as were all the patients and all visitors.

\subsection{Mass Gathering Warning}

The present COVID-19 pandemic [9] caused by a novel Corona virus has refocused global attention on national, regional, and pandemic spread through mass gatherings. It was decided to postpone the March Dajia Matsu Pilgrimage (from Mar 18 to Mar 28) to avoid the risk of community infection and to prevent potential chains of infection because it would be impossible to find the source and spread would be inevitable. A "social distancing" [10] guideline consistent with the WHO guidance on social distancing for COVID-19 $[11,12]$, has been established as a recommendation for the general public. The guidance suggested includes avoiding close social contact or gatherings such as exhibitions, sports competitions and concerts. If there is a need for close contact with others all persons are obliged to wear a medical mask. Unnecessary activities should not be held.

\section{Result}

Until now, the performance of Taiwan's COVID-19 epidemic infection control was ranked first place among the 23 OECD countries. Most of the confirmed patients were aged among 20 to 40 years old, which accounted for $60 \%$ of total confirmed cases, with a total mortality rate of about $1.4 \%$. The median time from the onset of symptoms to the release from isolation was 30 days.

\subsection{Containment of Immigration}

Containment of immigration from overseas has successfully reduced the spread of COVID-19 in the community. (See Figure 1) The satisfication rate from questionnaire about the process and quality of containment was very high.

\subsection{Hospital Clustering Infection}

Everyone who had been in the infected area was recalled for examination and two confirmed cases were found. One is the patients' family on the same floor and in different wards who have been discharged, and the other patient on the same floor and in different wards.

\subsection{Mass Gathering Warning}

During the Chingming Holiday, between April 02 and April 05, there was a huge flow of people into a number of major tourist attractions. From early morning on the first day there was heavy traffic on the national highways. Accommodation in the Kenting National Park reached 90\% of capacity and later the night markets in the middle and south of Taiwan were full of people returning home for shopping. After an emergency warning was sent out on the Taiwan NHCC and all these people were warned to keep the necessary social distance and wear masks, later they also had to stay inside their homes for 14 days. Due to this preventive action, there is no obvious increase in number of new confirmed cases of COVID-19 in Taiwan.

\section{Discussion}

\subsection{The Importance of Testing People in the High-risk Groups, Disaster Mitigation Model and Avoidance of Mass Gathering}

Although containment of immigration from overseas has successfully reduced the spread of COVID-19 in the community, however, significantly more cases exist than have been found and protection against this second COVID-19 wave should be expanded. This should be done by testing more people in the high-risk groups such as personnel in essential services, medical staff, convenience store clerks, taxi drivers and others in frequent social contact. So far Taiwan is not facing the degree of catastrophe such as that of Europe or the USA and general testing would be an unnecessary strain on resources.

In the hospital clustering infection, Taiwan $\mathrm{CDC}$ will control the epidemic situation should any similar event happen in the future and the whole hospital will not be closed. Disaster mitigation model will be performed by closing the whole building in the hospital, preventing new patients from being admitted to the same building, and placing all the inpatients to the other designated area. All existing patients will be tested and the medical staff will be isolated.

From the experience of Japan, as we know, March 20 to March 22 is Japan's three-day holiday (Cherry Blossom Festival), just in time to meet the annual cherry blossom season in Japan, and there are a large numbers of cherry scenic spots. The average number of new confirmed cases of COVID-19 per day during that week was about 40. Ten days after Cherry Blossom Festival, the number of newly diagnosed cases in Japan has increased sharply to 200 per day, which is five times higher than that before Festival. Taking a look at the other side of the Pacific Ocean, most of the spring holidays in american universities are in early March, which was accompanied with the epidemic outbreak in the United States since late March. It is coincidence that human flows provide excellent conditions for the spread of infectious transmitted diseases.

17 years ago in Taiwan, before the SARS epidemic, it was also the same condition with the Chingming holiday (from April 02 to April 05). Three weeks after the Chingming holiday in 2003, TMHH was closed on April 24, although the real epidemic peak was in the week of May 20. Therefore, this year in Taiwan, during the Chingming Holiday, between April 02 and April 05, there was also a huge flow of people into a number of major tourist attractions. After an emergency warning was sent out on the Taiwan NHCC and all these people were warned to keep the necessary social distance and wear masks, later they also had to stay inside their homes for 14 days. Due to this 
preventive action, there is no obvious increase in number of new confirmed cases of COVID-19 in Taiwan. Obviously, an emergency warning for mass gathering, keep the necessary social distance and wear masks could reduce the spread of COVID-19.

\subsection{Economic Impact}

Due to this severe economic impact from COVID-19 epidemic, which affecting many industries and corporations, Taiwan government quickly responded with the three major principles of "epidemic prevention, bail-out and revitalization". The impacted industries and businesses also proposed a bail-out plan based on the four principles of "rain and dew with even distribution, immediate good outcome, consolidating foundation with resilience, and accelerated public construction". Taiwan government announced the "Severe Special Infectiousness" on February 25, 2020. with the Special Regulations on Pneumonia Prevention and Relief and Revitalization (hereinafter referred to as the Special Regulations) serve as the legal basis for conducting epidemic prevention activities and raising funds. According to this special regulations, Taiwan government has set out the first additional budget of 20 billion USD, with existing budget and funds of nearly 13.33 billion USD to form a total of 33.33 billion USD for investment to rescue and revitalize the industries and corporations. Furthermore, the special regulations were revised to expand the special budget by additional 50 billion USD, for reducing personal taxes and increasing household expenses to industrial bailouts. Besides, Taiwan government overweights to send an additional loan with 233.33 billion USD to the industries and corporations.

The impact of this COVID-19 epidemic on the economy was originally only expected to be on manufacturing from China. Now, the epidemic in Europe and the United States is far more serious than initially expected, and the global recessing demand is more uncertain, which making Taiwan's industry was pessimistic about the first half of 2020. However, the COVID-19 epidemic may change human lifestyles, including daily social, working, shopping, communication and entertainment etc., and will generate novel or innovative business opportunities including biotechnological medical industry, artificial intelligence, 5G, internet of things etc.. Taiwan government will take the "thinking of headquarter economy" as a guide to Taiwan's global layout and industrial restructuring, and encourage Taiwan's businesses to keep core value activities such as operational big data analysis and decision-making in Taiwan.

\section{Conclusion}

Because of sound public health and healthcare systems, combined with public acceptance of protective policies influenced by the 2003 SARS outbreak, Taiwan effectively delayed and contained community transmission in the COVID-19 outbreak. As the global pandemic outbreak continues and economic recession gradually appears, Taiwan will still need to evaluate associated proactive policy decisions to sustain the system especially by innovative technology such as telemedicine, teleeducation, and teleoffice etc. A further integrated approach that incorporates ministries such as Health and Welfare, Economy, Transport, Technology, and Interior affairs can increase resilience and better prepare nations for future events.

\section{Acknowledgements}

We thank the members of Taiwan CDC, Central Epidemic Command Center expert team, and partnering departments who have worked tirelessly since the beginning of the COVID-19 pandemic.

\section{References}

[1] Córdova-Lepe F, Gutiérrez-Aguilar R, Gutiérrez-Jara JP. Number of COVID-19 cases in Chile at 120 days with data at 21/03/2020 and threshold of daily effort to flatten the epi-curve. Medwave. 2020 Mar 27; 20 (2): e7861. doi: 10.5867/medwave.2020.02.7861.

[2] Lin C, Braund WE, Auerbach J, Chou J-H, Teng J-H, Tu P, et al Policy decisions and use of information technology to fight 2019 novel coronavirus disease, Taiwan. Emerg Infect Dis. $2020 \mathrm{Jul}$. https://doi.org/10.3201/eid2607.200574.

[3] Matthew JA, Anne C. S, and Josiah D. R. Flattening the Curve for Incarcerated Populations - Covid-19 in Jails and Prisons. N Engl J Med. 2020 Apr. DOI: 10.1056/NEJMp2005687.

[4] James Griffiths. Taiwan's coronavirus response is among the best globally. CNN World. 2020 Apr. 04 https://www.cnn.com/2020/04/04/asia/taiwan-coronavirus-res ponse-who-intl-hnk/index.html.

[5] Rothe C, Schunk M, Sothmann P, et al. Transmission of 2019-nCoV infection from an asymptomatic contact in Germany. N Engl J Med 2020; 382: 970-1.

[6] Bai Y, Yao L, Wei T, et al. Presumed asymptomatic carrier transmission of COVID-19. JAMA 2020 February 21 (Epub ahead of print).

[7] Li R, Pei S, Chen B, et al. Substantial undocumented infection facilitates the rapid dissemination of novel coronavirus (SARS-CoV2). Science 2020 March 16 (Epub ahead of print).

[8] Hao-Yuan Cheng, Shu-Wan Jian, Ding-Ping Liu, Ta-Chou $\mathrm{Ng}$, Wan-Ting Huang, Hsien-Ho Lin. Contact Tracing Assessment of COVID-19 Transmission Dynamics in Taiwan and Risk at Different Exposure Periods Before and After Symptom Onset. JAMA Intern Med. doi: 10.1001/jamainternmed.2020.2020.

[9] WHO. Coronavirus disease (COVID-19) outbreak. 2020. https://www.who.int/emergencies/diseases/novel-coronavirus2019 (accessed March 17, 2020).

[10] Social distance precaution guideline on response to COVID-19 outbreak.

https://www.cdc.gov.tw/File/Get/qhvoUn6aFreGPD8e4B5Gk Q. 
[11] WHO. Key planning recommendations for mass gatherings in the context of the current COVID-19 outbreak. Interim guidance. $\quad$ Feb 2020 . https://apps.who.int/iris/bitstream/handle/10665/331004/WHO -2019-nCoV-POEmassgathering-2020.1-eng.pdf?sequence $=1$ \&isAllowed $=y($ accessed March 17, 2020).
[12] WHO. Coronavirus diseases (COVID-19) advice for the public. 2020.

https://www.who.int/emergencies/diseases/novel-coronavirus2019/advice-for-public (accessed March 18, 2020). 